\title{
MEASURABLE MULTIFUNCTIONS AND THEIR APPLICATIONS TO CONVEX INTEGRAL FUNCTIONALS
}

\author{
NIKOLAOS S. PAPAGEORGIOU
}

University of California

1015 Department of Mathematics

Davis, California 95616

(Received June 6, 1988 and in revised form September 26, 1988)

\begin{abstract}
The purpose of this paper is to establish some new properties of set valued measurable functions and of their sets of integrable selectors and to use them to study convex integral functionals defined on Lebesgue-Bochner spaces. In this process we also obtain a characterization of separable dual Banach spaces using multifunctions and we present some generalizations of the classical "bang-bang" principle to infinite dimensional linear control systems with time dependent control constraints.
\end{abstract}

KEY WORD AND PHRASES. Measurable multifunction, integrably bounded, measurable selection, Souslin space, Weak Randon-Nikodym property, set valued conditional expectation, infinite dimensional linear control systems, bang-bang principle, convex normal Integrand, subgradient, subdifferential, confugate, effective domain, absolutely continuous functional, singular functional, Yosida-Hewitt theorem. 1980 AMS SUBJECT CLASSIFICATION CODES. Primary 28A45, 46G10, 46E30, Secondary 5400.

\section{INTRODUCTION.}

In the last decade the study of measurable set valued functions has been developed extensively, both in the theoretical direction and the direction of applications. Many mathematicians have contributed significant results in this area, which combines challenging theoretical problems with 1mportant applications in a variety of fields, like optimization theory, optimal control, statistics and mathematical economics. In all those areas the systematic use of multifunctions has allowed people to make significant progress and solve many problems.

With a series of recent papers [18] + [25] the author has started an effort to extend the general theory of Banach space valued multifunctions and the closely related theory of multimeasures. The present paper continues this effort and provides some applications of the theoretical results obtained.

Briefly this paper is organized as follows. In the next section we establish our notation and for the convenience of the reader we recall some basic definitions and 
facts from the general theory of multifunctions and the theory of measurable integrands. In section 3 we have gathered some results, in which starting from properties of the set of integrable selectors of a multifunction we extract information about its pointwise properties, the structure of its conditional expectation and the properties of the underlying Banach space. Some other related observations of functional analytic nature are also included. In section 4, we proceed to a detailed study of the properties of the set of integrable selectors of a multifunction and we present an application to control theory ("bang-bang" type results). Finally in section 5, we use the results obtained earlier in order to study convex integral functionals that appear often in problems of optimization, optimal control and mathematical economics. With this combination of theoretical and applied results, we want to emphasize the importance and the versatility of the theory of multifunctions and attract the interest of mathematicians from different areas.

\section{PREL IMINARIES.}

Let $(\Omega, \Sigma)$ be a measurable space and $X$ a separable Banach space. Throughout this work we will be using the following notations:

$$
\begin{aligned}
& P_{f(c)}=\{A \underline{C} X: \text { nonempty, closed, (convex) }\} \\
& P_{(w) k(c)}(X)=\{A \subseteq X: \text { nonempty, (w-) compact, (convex) }\}
\end{aligned}
$$

Also we will be using the following additional three pieces of notation. Let

$$
A \varepsilon 2^{X}-\{\emptyset\} . \text { By }|A| \text { we will denote the norm of } A \text { i.e., }|A|=\sup _{\alpha \varepsilon A}|| \alpha|| \text {, by }
$$$$
\sigma(., A) \text { the support function of } A \text { i.e., } \sigma\left(x^{*}, A\right)=\sup _{\alpha \varepsilon A}\left(x^{*}, \alpha\right), x^{*} \varepsilon X^{*} \text { and by } d(\bullet, A) \text { the }
$$
distance function from $A$ i.e., $d(x, A)=\underset{\alpha \varepsilon A}{\inf }|| x-\alpha \|$.

A multifunction $F: \Omega \rightarrow P_{f}(X)$ is said to be measurable if for every $x \in X$, the function $\omega \rightarrow d(x, F(\omega))$ is measurable. This definition is equivalent to saying that there exist $f_{n}: \Omega+x$ measurable functions s.t. for every $\omega \varepsilon \Omega$

$F(\omega)=c\left\{_{n}(\omega)\right\}_{n \geqslant 1}$ ("Castaing's representation" - see Castaing - Valadier [5]).

A function $f: s \rightarrow X$ s.t. $f(\omega) \varepsilon F(\omega)$ is said to be a selector of $F(\cdot)$. The problem of existence of measurable selectors is central in the theory of multifunctions. In applications the most widely used selection theorem, is the following one which was first proved by Aumann [1] for Polish spaces and was later extended to Souslin spaces by Saint-Beuve [30]. By $\Sigma$ we will denote the universal $\sigma-f i e l d$ corresponding to $\Sigma$.

THEOREM 2.1 [30]. If $X$ is a Souslin space and $F: s \rightarrow 2^{X}-\{\emptyset\}$ is a multifunction

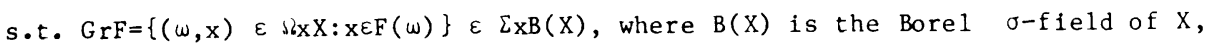
then there exist $f_{n}: s+X, \sum$-measurable selectors of $F(\cdot)$ s.t. $F(\omega) \subseteq c l\left\{f_{n}(\omega)\right\}_{n}>1$ for all wes? 
REMARKS. a) If $(\Omega, \Sigma, \mu)$ is a $\sigma$-finite complete measure space, then $\Sigma=\Sigma$.

b) Recall that a Souslin space is always separable, but it need be metrizable (for example a separable Banach space with the weak topology). c) If $F()$.18 closed valued and measurable in the sense defined earlier then GrF $\varepsilon \sum_{X B}(X)$ (graph measurablity). The converse is true if $\Sigma=\Sigma$ (i.e. $\Sigma$ is complete).

Let $(\Omega, \Sigma, \mu)$ be a $\sigma-f$ inite measure space and let $S_{F}^{1}=\left\{f(\cdot) \varepsilon L_{X}^{1}: f(\omega) \in F(\omega)\right.$. $\mu-a \cdot e\}$.

Using $S_{F}^{1}$ we can define a set valued integral for $F($.$) by$

$$
\int_{\Omega \Omega} F(\omega) d \mu(\omega)=\left\{\int_{\Omega} f(\omega) d \mu(\omega): f \varepsilon S_{F}^{1}\right\} \text {. We say that } F: \Omega \rightarrow P_{f}(X) \text { is integrably bounded }
$$

if it is measurable and $\omega \rightarrow|F(\omega)| \varepsilon L_{+}^{1}$. Using theorem 2.1 we can see that if $F(\cdot)$

is integrably bounded then $S_{F}^{1}$ and $\int_{\Omega} F$ are both nonempty.

Let $\mathrm{K} \subseteq \mathrm{L}_{\mathrm{X}}^{1}$ be nonempty. We say that $\mathrm{K}$ is decomposable (a lso known as "convex with respect to switching") If for all $A \varepsilon \Sigma$ and all $\left(f_{1}, f_{2}\right) \varepsilon K x K, x_{A} f_{1}+x_{A} c f_{2} \varepsilon K$. In [9], theorem 3.1., Hial-Umegak1 proved that if $\mathrm{K}$ is closed and decomposable, then there exists $F: \Omega \rightarrow P_{f}(X)$ measurable s.t. $K=S_{F}^{1}$. Furthermore if $K$ is bounded, then $F($.$) is integrably bounded. Using this fact, Hiai-Umegaki [9], went on and defined as$ set valued conditional expectation for $F($.$) as follows. Let to be a$ sub- $\sigma-f i e l d$ of $\Sigma$ let $F: \Omega \rightarrow \mathrm{P}_{\mathrm{f}}(\mathrm{X})$ be measurable with $\mathrm{S}_{\mathrm{F}}^{1} \neq \emptyset$ Define

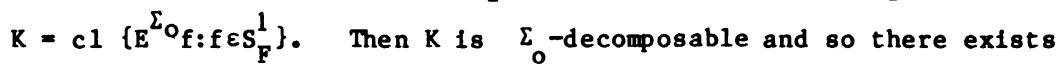

$$
E^{\Sigma} o_{F: \Omega}+P_{f}(X) \Sigma_{0} \text { measurable s.t. } K=S_{E}^{1} \Sigma_{O_{F}} \text {. The multifunction } E^{\Sigma} o_{F(.)} \text { is the }
$$
set valued conditional expectation of $F($.$) .$

Next let $(\Omega, \Sigma, \mu)$ be a complete, $\sigma-f$ inite measure space and $X$ a separable Banach space. Let $f: \Omega x X+R U\{+\infty\}, f \neq+\infty$. Following Rockafellar [28] we say that $f(.,$.$) is$ a normal integrand if the following conditions are satisfied:

1) $(\omega, x) \rightarrow f(\omega, x)$ is $\sum x B(x)$ measurable

1i) for all $\omega \varepsilon \Omega, x \rightarrow f(\omega, x)$ is lower seml continuous $(1.8 \cdot c)$

Using the celebrated "Von Neumann projection theorem" (see Castaing - Valadier [5], theorem III - 23) we can show that the above two conditions are equivalent to the following. Recall that if $g: X \rightarrow R U\{+\infty\}$, then epig $=\{(x, \lambda) \varepsilon X x R: f(x)<\lambda\}$.

$\left.1^{\prime}\right)$ the multifunction $\omega+\operatorname{epif}(\omega,$.$) is graph measurable$

1i') for all $\omega \varepsilon \Omega$, epif( $\omega,.) \varepsilon P_{f}(X \times R)$

Note that 11') immediately implies that $\omega+\operatorname{epif}(\omega,$.$) is measurable. Because of$ condition 1) a normal integrand $f(.,$.$) is superpositionally measurable 1.e. if$ $x: \Omega \rightarrow X$ is measurable, then $\omega \rightarrow f(\omega, x(\omega))$ is measurable. A well known example of normal integrands are the Caratheodory integrands. A normal integrand $f(.,$.$) is said$ 
to be convex, if for all $\omega \varepsilon \Omega, f(\omega,$.$) is convex.$

Let us also recall some notions from convex analysis. Let $f \in \bar{R}^{-X}$. We define $\partial f(x)=\left\{x^{*} \varepsilon X^{*}:\left(x^{*}, y-x\right)<f(y)-f(x)\right.$ for all $\left.y \in X\right\}$. This is called the subdifferential of $f(\cdot)$ at $x$. Also we define $f^{\star} \varepsilon \bar{R}^{-X^{*}}$ by $f^{\star}\left(x^{\star}\right)=\sup \left\{\left(x^{\star}, x\right)-f(x) ; x \in X\right\}$ and this is known as the conjugate of $f(\cdot)$. The conjugate and the subdifferential are related by the Young-Fenchel equality, namely: $x^{\star} \varepsilon \partial f(x)$ if and only if $f^{\star}\left(x^{\star}\right)+f(x)=$ $\left(x^{\star}, x\right)$. Since we are dealing here with extended real valued functions we define the effective domain of $f$ to be: domf $=\{x \in X: f(x)<\infty\}$.

Finally, recall that $X$ has the weak Randon-Nikodym property (WRNP) if it has the Radon-Nikodym property for the Pettis integral.

\section{MEASURABLE MULTIFUNCTIONS.}

We start with a result in which, knowing the structure of the set of integrable selectors of a multifunction, we deduce some pointwise properties of the multifunction. Another such result was obtained by the author in [25] (theorem 5.1).

Assume that $(\Omega, \Sigma, \mu)$ is a complete, $\sigma$-finite measure space and $X$ a weakly sequentially complete, separable Banach space.

THEOREM 3.1. If $X^{*}$ has the WRNP and $S_{F}^{1}$ nonempty, bounded, closed and convex.

$$
\text { then } F(\omega) \varepsilon P_{w k c}(X) \mu-a . e .
$$

PROOF. From Hi ai-Umegaki [9] (theorem 3.2) we know that $F($.$) is integrably$ bounded and so for all $\omega \varepsilon \Omega \cdot N, \mu(N)=0, F(\omega)$ is bounded.

Suppose that for some $\omega \varepsilon \Omega-N, F(\omega)$ is not w-compact. Then the Eberlein-Smulian theorem and the fact that $X$ is w-sequentially complete give us a sequence $\left\{x_{n}\right\}_{n>1}$ with no Cauchy subsequence. Recalling that $\left\{x_{n}\right\}_{n \geqslant 1}$ is bounded, we can apply the result of Rosenthal [29] and deduce that $\left\{x_{n}\right\}_{n \geqslant 1}$ is an $\ell^{1}$-sequence. Hence $\ell^{l} C X$ a contradiction to the fact that $X^{*}$ has the WRNP (see Musial-Ryll Nardzewski [17]).

REMARK. If $X *$ has the $R N P$, then the result is immediate, since $X$ is reflexive (see Diestel -Uh1 [7], corollary 11, p. 198). But we know that in general the WRNP does not imply the RNP.

In fact, when $X$ is a Banach lattice we can have a partial converse of the above theorem. So assume that $(\Omega, \Sigma, \mu)$ is a complete, $\sigma-f$ inite measure space and $X$ a separable Banach lattice.

THEOREM 3.2. If the following implication holds: " $\mathrm{s}_{\mathrm{F}}^{1}$ is nonempty, convex and $w$ compact $==>F(\omega) \in P_{\text {wkc }}(X) \mu-a . e . "$, then $X *$ is separable and $w$-sequentially complete.

PROOF. We will show that $\ell_{1} 4 \mathrm{x}$. Suppose not. Then from our hypothesis if

$\mathrm{S}_{\mathrm{F}}^{1} \underline{\mathrm{C}} \mathrm{L}_{\ell^{1}}^{1}(\Omega)$ is nonempty, convex and w-compact then $F(\omega) \varepsilon \mathrm{P}_{\text {wkc }}\left(\ell^{1}\right)$ wra.e. Set $M(A)$

$=\left\{\int_{A} f(\omega) d \mu(\omega): f \varepsilon S_{F}^{1}\right\}, A \varepsilon \Sigma$. Then $M(\cdot)$ is a $P_{\text {wkc }}\left(\ell^{1}\right)$-valued multimeasure with a 
$P_{\text {wkc }}\left(\ell^{1}\right)$-valued density, a contradiction to example 2 of costé [6]. So $\ell_{1} 4$ x and

then from a result of Lotz (see Diestel-Uhl [7], p.95) we deduce that $x^{*}$ has the RNP. Since $X$ is separable from corollary 8, p.98 of Diestel-Uhl [7], we deduce that $X^{\star}$ is separable. Also $c_{0} 4^{4} X^{*}$ and so theorem $1, c_{.4}$ of Lindenstrauss - Tzafrir1 [16] tells us that $X^{*}$ is w-sequentially complete. S.E.D.

Also we have a weak compactness result for the set of integrable selectors of a multifunction. Another such result can be found in [25]. But first we will need a property of decomposable subsets of $L_{X}^{1}$, which was proved by the author in [26] (Proposition 5.1 - see also Dieste1-Uh1 [7], Theorem 4, p. 104). For the convenience of the reader we recall the result here. Let $(\Omega, \Sigma, \mu)$ be a $\sigma$-finite measure space and $X$ a Banach space.

PROPOSITION 3.1 [26]. If $K \underline{C} L_{X}^{1}$ is nonempty, decomposable and bounded then $K$ is uniformly integrable.

Now assume that in addition $X$ is weakly sequentially complete.

THEOREM 3.3. If $\mathrm{K} \underline{\mathrm{C}} \mathrm{L}_{\mathrm{X}}^{1}$ is nonempty, decomposable, bounded and w-closed, with no $\ell^{1}$-sequence then $\mathrm{K}$ is $\mathrm{w}$-compact.

PROOF. From Proposition 4.1 we know that $K$ is untformly integrable. Also since $X$ is weakly sequentially complete, so is $L_{X}^{1}$ (see Talagrand [31]). Combining these facts with Corollary 8 of Bourgain [4] and the Eberlein-Smullan theorem we get that $K$ is w-comp act.

Q.E.D.

REMARK. If $X$ is separable then $K=S_{F}^{1}$ where $F: \Omega \rightarrow P_{f}(X)$ is integrably bounded. So indeed our result is a w-compactiess result for the set of integrable selectors of a multifunction.

4. PROPERTIES OF THE SET OF INTEGRABLE SELECTORS.

This section is devoted to a detailed study of the properties of the set of integrable selectors of measurable (or graph measurable) multifunctions. Those results are then applied to the analysis of a family of infinite dimensional linear control systems with time dependent control constraints. The material of this section will also be used in the next section, in the study of convex integral functionals.

We will start with an auxilliary result that we will need in what follows and which also generalizes Theorem 1.5 of Hiai-Umegak1 [9].

Assume that $(\Omega, \Sigma, \mu)$ is a complete, $\sigma$-finite measure space and $X$ a separable Banach space.

LEMMA A. If $F: \Omega+2^{X}-\{\varnothing\}$ is graph measurable and $s_{F}^{1} \neq \emptyset$ then $\overline{\operatorname{conv}} s_{F}^{1}=s^{1} \frac{\operatorname{conv}}{F}$ PROOF. Clearly $\overline{\operatorname{conv}} \mathrm{S}_{\mathrm{F}}^{1} \underline{\mathrm{C}} \mathrm{S}^{1} \overline{\operatorname{conv}} \mathrm{F}$. Suppose that the inclusion 1s strict so we can find $f \in S^{1} \overline{\operatorname{conv}} F$ s.t. $f \neq \overline{\operatorname{conv}} S_{F}^{1}$. From the strong separation theorem there exists $u(.) \varepsilon L_{X^{*} w^{*}}^{\infty}=\left[L_{X}^{1}\right]^{*}$ s.t. $\sigma\left(u, \overline{\operatorname{convS}}{ }_{F}^{1}\right)\langle\langle u, f\rangle$

But from [23] we know that: 


$$
\begin{gathered}
\sigma\left(u, \overline{\operatorname{conv}}_{F}^{1}\right)=\sigma\left(u, S_{F}^{1}\right)=\sup _{h \in S_{F}} \int_{\Omega}(u(\omega), h(\omega)) d \mu(\omega) \\
\int_{\Omega} \sup _{x \in F(\omega)}(u(\omega), x) d \mu(\omega)=\int_{\Omega} \sigma(u(\omega), F(\omega)) d \mu(\omega)
\end{gathered}
$$

Hence $\left.\sigma\left(u, \overline{\operatorname{convs}} S_{F}^{1}\right)<\int_{\Omega} u(\omega), f(\omega)\right) d \mu(\omega)$

On the other hand since $f(.) \varepsilon S^{1} \overline{\text { convF }}$, we have $f(\omega) \overline{\varepsilon \operatorname{conv}} F(\omega) \mu$-a.e.

$$
(u(\omega), f(\omega))<\sigma(u(\omega), F(\omega)) \mu-a \cdot e=\Rightarrow \int_{\Omega}(u(\omega), f(\omega)) d \mu(\omega)<\int_{\Omega} \sigma(u(\omega), F(\omega)) d \mu(\omega) \Rightarrow
$$

a contradiction.

Using this lemma we can have the following Lyapunov type result. So we assume that $(\Omega, \Sigma, \mu)$ is a complete, $\sigma-f$ inite, nonatomic measure space and $X$ a separable Banach space.

THEOREM 4.1. If $F: \Omega \rightarrow 2^{X}-\{\emptyset\}$ is graph measurable and $S_{F}^{1} \neq \emptyset$ then $\overrightarrow{s_{F}^{1}}=S^{1} \frac{c_{\text {conv }}}{F}$ (here $w$ indicates the weak topology on $L_{X}^{1}$ ).

PROOF. Note that $s^{1} \overline{\text { convF }}$ is a closed, convex set. Hence we immediately have:

$$
\overline{s_{F}^{1}} \quad \underline{c} \frac{1}{\operatorname{convF}}=\overline{\operatorname{conv}} s_{F}^{1}(1 \text { emma a) }
$$
Next let $g \varepsilon \quad \operatorname{conv} S_{F}^{1}, g=\sum_{i=1}^{n} \lambda_{i} f_{i}, f_{1} \varepsilon S_{F}^{1}$. Let $v\left(g,\left\{u_{k}\right\}_{k=1}^{m}, \varepsilon\right\}$ be a weak
neighborhood of $g$. So

$$
V=\left\{h \varepsilon L_{X}^{1}:\left|\left\langle u_{k}, g-h\right\rangle\right|\langle\varepsilon, k=1, \ldots, m\}\right.
$$

where $u_{k} \varepsilon L_{X^{*}}^{\infty}$ and $\varepsilon>0$. Let $L: L_{X}^{1} \rightarrow R^{m}$ be defined by

$$
L(h)=\left\langle u_{k}, h\right\rangle_{k=1}^{m}
$$

Clearly $L(\cdot)$ is a continuous linear operator. We claim that $L\left(S_{F}^{1}\right)$ is convex. Let $f_{1}, f_{2} \varepsilon K$ and consider $A \rightarrow m(A)=L\left(x_{A}\left(f_{1}-f_{2}\right)\right)$. It is easy to see that $m(\cdot)$ is a vector measure of bounded variation which is $\mu$-continuous. So applying Lyapunov's theorem we get that Range(m) is

convex $\Rightarrow \quad \underset{A E \Sigma}{U} L\left(x_{A}\left(f_{1}-f_{2}\right)\right)+L\left(f_{2}\right)={ }_{A E \Sigma}^{U} L\left(x_{A} f_{1}+x_{A} c f_{2}\right)$

is convex. But since $\mathrm{K}$ is decomposable.

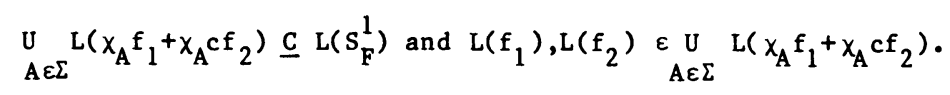

So indeed $L\left(S_{F}^{1}\right)$ is convex.

Thus we can find $f \varepsilon S_{F}^{1}$ s.t. 


$$
L(g)=\left\{\sum_{i=1}^{n} \lambda_{i}\left\langle u_{k}, f_{i}\right\rangle\right\}_{k=1}^{m}=L(f) \text {. }
$$

Therefore $v\left(g,\left\{u_{k}\right\}_{k=1}^{m}, \varepsilon\right\} \cap s_{F}^{1} \neq \emptyset$ and so.

$$
\overline{s_{F}^{1}} \overline{\operatorname{conv}} s_{F}^{1}
$$

Combining (4.1) and $(4.2)$ we finally have:

$$
{\overline{s_{F}^{1}}}^{w}=\overline{\operatorname{conv}} s_{F}^{1}
$$

An immediate important consequence of theorem 4.1 is the following result. The

spaces remain as before.
THEORM 4.2 . If $F: \Omega \rightarrow P_{w k c}(X)$ is measurable and $S_{F}^{1}, s_{\text {extF }}^{1} \neq \emptyset$ then $S_{\text {extF }}^{1}=s_{F}^{1}$

PROOF. From Benamara [2] we know that $\omega \rightarrow \operatorname{extF}(\omega)$ is graph measurable. So applying Theorem 4.1 we get that $\overline{s_{\text {extF }}^{1}}=s^{1} \frac{1}{\text { convextF }}=S_{F}^{1}$ (Krein-Milman theorem).

Another interesting consequence is the following result about the set valued

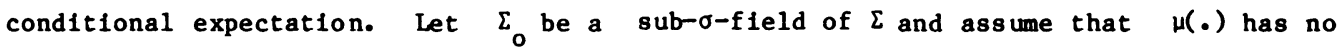
$\Sigma_{\text {o }}$-atoms.

THEOREM 4.3. If $F: \Omega \rightarrow P_{F}(X)$ is integrably bounded and $S_{F}^{1}$ is w-compact in $L_{X}^{1}$ then $E^{\Sigma}{ } F(\omega)$ is $\mu-$ a.e. convex.

PROOF, From Theorem 4.1 we have that $\cdot \overline{\mathrm{s}^{1} \Sigma_{\mathrm{O}_{F}}}$ is convex. Since

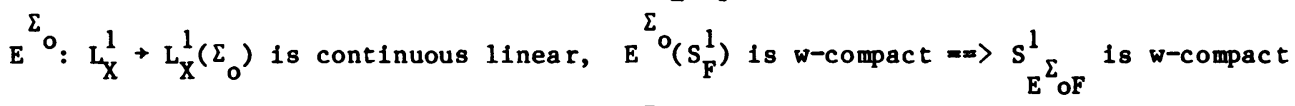

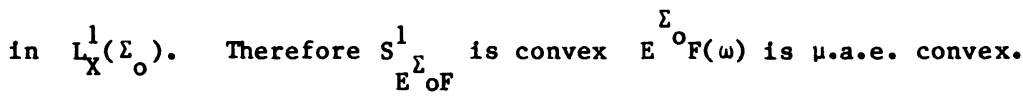

Now we examine the strong closure of $S_{F}^{1}$. So let $(\Omega, \Sigma, \mu)$ is a complete, $\sigma-f$ inite measure space and $X$ a separable Banach space.

THEOREM 4.4. If $F: \Omega \rightarrow 2^{X} \backslash\{\emptyset\}$ is graph measurable and $s_{F}^{1} \neq \emptyset$ then $s_{F}^{\overline{1}}=s_{\bar{F}}^{1}$

PROOF. Since $S_{F}^{1}$ is closed in $L_{X}^{1}$, we get that $\overline{S_{F}^{1}} \underline{c} S_{F}^{1}$. Let $f(.) \varepsilon S_{\bar{F}}^{1}$ For $\mathrm{n}>1$ let $\mathrm{L}_{\mathrm{n}}: \Omega \rightarrow 2^{\mathrm{X}}-\{\varnothing\}$ be defined by:

$$
\begin{aligned}
& L_{n}(\omega)=\left\{x \in F(\omega):|| x-f(\omega) \|<\frac{1}{n}\right\} \\
& G r L_{n}=\left\{(\omega, x) \varepsilon \Omega \& X:|| x-f(\omega) \mid<\frac{1}{n}\right\} \cap G r F
\end{aligned}
$$

Note that $(\omega, x) \rightarrow \| x-f(\omega)||$ is a Caratheodory function and so it is jointly 
measurable. Hence $\left\{(\omega, x) \in \Omega\left(a x X:\|x-f(\omega)\|<\frac{1}{n}\right\} \varepsilon \sum x B(X)\right.$. Also by hypothesis $\operatorname{GrF} \varepsilon \Sigma_{x B}(X)$. Therefore for all $n \geq 1, \operatorname{GrL}_{n} \varepsilon \sum \times B(X)$. Apply Theorem 2.1 to find $f_{n}: \Omega \rightarrow X$ measurable s.t. $f_{n}(\omega) \varepsilon L_{n}(\omega)$ for all $\omega \varepsilon \Omega, n>1$. Then clearly for all $\omega E \delta, f_{n}(\omega) \$ f(\omega)$. Since $\quad\left\|f_{n}(\omega)\right\|<\|f(\omega)\|+1$, applying the dominated convergence theorem, we get that

$$
f_{n} \stackrel{s-L_{X}^{\prime}}{\longrightarrow} f=\Rightarrow f S_{F}^{1} \Rightarrow \overline{s_{F}^{1}}=S_{F}^{1} .
$$

Q.E.D.

The result has an interesting consequence. However before passing to $1 t$, we need to have the following lemma. It generalizes a similar result of Hial-Umegaki [9], who required the multifunctions to be closed valued (see Corollary 1.2 of [9]). The spaces remain as before.

LEMMA B. If $F_{1}, F_{2}: \Omega \rightarrow 2^{X}-\{\emptyset\}$ are graph measurable and $S_{F_{1}}^{1}=S_{F_{2}}^{1} \neq \emptyset$ then $F_{1}(\omega)=F_{2}(\omega) \mu-$ a.e.

PROOF. Suppose not. Then there exists $A E \Sigma$ with $\mu(A)>0$ s.t. $F_{1}(\omega) F_{2}(\omega) \neq 0$ for all $\omega \varepsilon A$. Let $R: A \rightarrow 2^{X}-\{0\}$ be defined by $R(\omega)=F_{1}(\omega) F_{2}(\omega)$. Then $\operatorname{GrR} \varepsilon \Sigma_{A} x B(X)$ (where $\Sigma_{A}=\Sigma \quad A$ ). Apply theorem 2.1. to find $g: A \rightarrow X$ measurable s.t. $g(\omega) \varepsilon R(\omega)$ for all $\omega \varepsilon A$. Let $\left\{\Omega_{n}\right\}_{n>1}$ be a $\Sigma$-partition of $\Omega$ s.t. $\mu\left(\Omega_{n}\right)<\infty$. Define

$$
C_{m n}=\{\omega \varepsilon A: m-1<\|g(\omega)\|<m\} \quad \Omega_{n}
$$

Then clearly $\left\{C_{m n}\right\}_{m, n>1}$ is a $\Sigma$-partition of A. Since $\mu(A)>0$ we can

find $m, n \geqslant 1$ s.t. $\mu\left(C_{m n}\right)>0$. Set

$$
f(\omega)=\left\{\begin{array}{l}
g(\omega) \text { for } \omega C_{m n} \\
f(\omega) \text { for } \omega E \Omega^{\prime} C_{m n}
\end{array}\right.
$$

where $f(\cdot) E S_{F_{1}}^{1}$. Then because of the decomposability of $S_{F_{1}}^{1} f E S_{F_{1}}^{1}$, while $f \notin S_{F_{2}}^{1}$. This produces the derived contradiction.

Q.E.D.

Now we are ready for the theorem. in $\frac{1}{x}$

THEOREM 4.5. If $F: \Omega \rightarrow 2^{X} \cdot\{\phi\}$ is graph measurable and $S_{F}^{1}$ is nonempty and closed

$$
\text { then } F(\omega) \& P_{f}(X) \text { H-a.e. }
$$

PROOF. From Theorem 4.4 we have that $\overline{S_{F}^{1}}=S_{F}^{1}=S \frac{1}{F}$. Apply lemma $\beta$ to get that $F(\omega)=\bar{F}(\omega)$ H-a.e. $\Rightarrow F($.$) is valued \mu$-a.e.

Q.E.D.

Now we will apply the results of this section to obtain versions of the "bang-bang principle", for infinite dimensional linear control systems with time dependent control constraints. So consider the following system:

$$
\begin{aligned}
& \dot{x}(t)=A(t) x(t)+B(t) u(t) \\
& x(0)=x_{0}, u(t) \varepsilon U(t) a \cdot e, u(\cdot) \varepsilon L_{x}^{1}
\end{aligned}
$$


Here $t \varepsilon R_{+}, A(t)$ is an unbounded, linear operator and $B(.) \varepsilon L_{10 c}^{\infty}\left(R_{+} ; L(X)\right)$, where $L(X)$ is the space of all bounded linear operators from $X$ into itself. We will assume that the linear evolution problem $\dot{x}(t)=A(t) x(t), x(0)=x_{0}$ admits a fundamental solution $\phi:\{(t, s): 0<s<t\} \rightarrow L(X)$. Conditions on $A(\cdot)$ that guarantee the existence of $\Phi(.,$.$) can be found in Kato [12]. Then if B() u.(.) \varepsilon L_{10 c}^{1}\left(R_{1}, X\right)$, we know that (4.3) has a mild solution $x_{u}(\cdot) \varepsilon G_{X}\left(R_{+}\right)$given by:

$$
x_{u}(t)=\Phi(t, 0) x_{0}+\int_{0}^{t} \Phi(t, s) B(s) u(s) d s, t \varepsilon R_{+}
$$

Let $R(t)$ be the set of the attainable points of system (4.3) using all feasible controls and let $R_{e}(t)$ be the set of attainable points of (4.3) using extremal controls (i.e. $u(t) \varepsilon \operatorname{ext} U(t)$ a.e.).

The next theorem establishes the relation between those two sets and can be viewed as an infinite dimensional generalization of the classical "bang-bang principle" (see He rmes - LaSa1le [8]).

THEOREM 4.6. If $U: R_{+} \rightarrow R_{w k c}(X)$ is measurable and $s_{U}^{1}, s_{e x t U}^{1} \neq 0$

$$
\text { then for all } t \varepsilon R_{+}, \overline{R_{e}(t)^{w}}=\overline{R(t)}^{w}=\text { convex }
$$

PROOF. Clearly we need to show that $R(t) \underline{C} \overline{R_{e}(t)}$. So let $x \in R(t)$. By definition there exists $u(). E S_{U}^{1}$ s.t.

$$
x(t)=\Phi(t, 0) x_{0}+\int_{0}^{t} \Phi(t, s) B(s) u(s) d s
$$

From Theorem 4.2 we know that there exists net $u_{b}(\cdot) \in S_{\text {extU }}^{1}$ s.t. $u_{b} \stackrel{w-L_{X}^{1}}{\rightarrow}$. Then for every $x^{\star} \varepsilon X^{*}$ we have:

$$
\int_{0}^{t}\left(x^{*}, \Phi(t, s) B(s) u_{b}(s)\right) d s=\int_{0}^{t}\left(B^{*}(s) *(t, s) x^{*}, u_{b}(s) d s\right.
$$

Note that $\left\|B^{*}(s) \Phi *(t, s) x^{*}\right\|<\left\|B^{*}(s)\right\|\|\Phi *(t, s)\|\left\|x^{*}\right\|$. Also from the properties of $\Phi(.,$.$) (see Kato [12]), we have$ $\|\Phi(t, s)\|=\left\|{ }^{\prime}(t, s)\right\|<M$, while by hypothesis $\|B(s)\|=\|B *(s)\|$

$<|| B||_{L}^{\infty}[0, t]^{\circ}$

Hence $s \rightarrow B^{*}(s) \phi^{*}(t, s) X^{*}$ belongs in $L_{X^{*}}^{\infty}[0, t]$ and so we have:

$$
\begin{aligned}
& \int_{0}^{t}\left(B^{*}(s) \Phi *(t, s) x^{*}, u_{b}(s)\right) d s+\int_{0}^{t}\left(B^{*}(s) \phi *(t, s) x^{*}, u(s)\right) d s \\
\Rightarrow \quad & =\int_{0}^{t}\left(x^{*}, \Phi(t, s) B(s) u(s)\right) d s \\
\Rightarrow & \int_{0}^{t} \Phi(t, s) B(s) u_{b}(s) d s+\int_{0}^{t} \Phi(t, s) B(s) u_{b}(s) d s \\
& \operatorname{Set} x_{b}=\Phi(t, 0) x_{0}+\int_{0}^{t} \Phi(t, s) B(s) u(s) d s
\end{aligned}
$$

Then

$$
\Rightarrow x_{b} x_{0} \text { Clearly } x_{b} \in R_{e}(t) \Rightarrow x(t) \overline{\varepsilon R_{e}(t)^{w}}=>\overline{R_{e}(t)^{w}}=\overline{R(t)^{w}} .
$$


The convexity of the set is clear from the convexity of the values of U(.). Q.E.D. With an $L_{10 c}^{1}\left(R_{+}\right)$- boundedness hypothesis on the control constraint multifunction

$u(\cdot)$, we can improve the conclusion of Theorem 4.6 .

THEOREM 4.7. If $U: R_{+} \rightarrow R_{w k c}(X)$ is measurable and $|U(\cdot)| \varepsilon L_{10 c}^{1}\left(R_{+}\right)$

$$
\text { then for all } t \varepsilon R_{+}, R(t)=\bar{R}_{e^{(t)^{W}} \varepsilon P} \text { wkc }(X) \text {. }
$$
PROOF. By definition $R(t)=\Phi(t, 0) x_{0}+\int_{0}^{t} \Phi(t, s) B(s) U(s) d s$.
Note that for all $x^{*} \varepsilon X^{*}$

$$
\sigma\left(x^{*}, \Phi(t, s) B(s) U(s)\right)=\sigma(B *(s) \Phi *(t, s) x *, U(s))
$$

Also since $U(\cdot)$ is $P_{\text {wkc }}(X)$-valued and measurable, $\left(s, z^{\star}\right) \rightarrow \sigma\left(z^{\star}, u(s)\right)$ is a Caratheodory function from $\Omega X^{*}$ into $R$. Therefore it is jointly measurable and so $s \rightarrow \sigma\left(B^{*}(s) \phi^{*}(t, s) x^{*}, U(s)\right)$ is measurable. Invoking theorem III-37 of Castaing Valadier [5], we conclude that $s \rightarrow \Phi(t, s) B(s) U(s)$ is a $P_{w k c}(X)$-valued, integrably bounded multifunction. So we can apply proposition 3.1 of [18] (see also [22]) and get that

$$
\int_{0}^{t} \Phi(t, s) B(s) U(s) d s \in P_{w k c}(X) \Rightarrow R(t) \varepsilon P_{w k c}(X) t \varepsilon R_{+} \cdot
$$

Q.E.D.

When $X$ is a finite dimensilonal Banach space, we obtain an extension of LaSalle's "bang-bang principle" (see He rmes-LaSa1le [8]), to linear control systems with time dependent, nonconvex control constraints. Recall that if $F: \Omega \rightarrow 2^{X}$ - $\{\varnothing\}$ is graph measurable, $S_{F}^{1} \neq \emptyset$ and $\mu(\cdot)$ is nonatomic, then $\int_{\Omega} F$ is convex (see Klein-Thompson [13],

Theorem 17.1.6 and for a generalization to Banach spaces [23]).

THEOREM 4.8. If $U: R_{+}+P_{f}(X)$ is measurable and $|U(\cdot)| \varepsilon L_{10 c}^{1}\left(R_{+}\right)$

then for all $t \in R_{+}, \overline{R_{e}(t)}=R(t) \in P_{k c}(X)$.

5. CONVEX INTEGRAL FUNCTIONALS ON LEBESGUE-BOCHNER SPACES.

In this section we use the theoretical results obtained previously, to conduct a study of convex integral functionals which are defined on Lebesgue-Bochner spaces. Our work in this section extends earlier results of Rockafellar [27] (finite dimensions) and Bismut [3] (finite dimensional or separable, reflexive Banach spaces).

It is we1l known that if $X$ is finite dimensional space and an integral functional is weakly lower semicontinuous on $L_{X}^{1}$, then the integrand is automatically convex in the state variable (See Bi smut [3], Theorem 1 and Rockafellar [27] Theorem 1). He re we extend this result to separable Banach spaces and we present a different, simpler proof using Theorem 4.1 .

First we need a Lemma. As sume that $(\Omega, \Sigma, \mu)$ is a complete, $\sigma-f$ inite, nonatomic measure space and $X$ a separable Banach space.

LEMMA A. If $\mathrm{F}: \Omega \_\rightarrow 2^{\mathrm{X}}-\{\varnothing\}$ is graph measurable and $\mathrm{S}_{\mathrm{F}}^{1} \neq 0$, 
then $S_{F}^{1}$ is $w$-closed if an only if $F(\omega) \varepsilon P_{f c}(X) \mu-a . e$.

PROOF. First assume that $s_{F}^{1}$ is w-closed in $L_{X}^{1}$. Then from Theorem 4.1 . we
have $s_{F}^{1}=s^{1} \frac{1}{\text { convF }}$ Using Lemma $B$ of section 4 we conclude that $F(\omega)=\overline{\operatorname{convF}}(\omega) \mu-a_{.} e_{.} \Rightarrow F(\omega) \varepsilon P_{f c}(X) \mu-a_{.} e$. closed.

Now assume that $F(\omega) \varepsilon P_{f c}(X)$ wa.e. Then $S_{F}^{1}$ is closed and convex. So it is $w$

Now we are ready for our theorem. The spaces are as above.

Q.E.D.

Also if $f: \delta 3 x \rightarrow \bar{R}$ is an integrand for $x: \Omega \rightarrow X$ measurable we

set $I_{f}(x)=\int_{\Omega} f(\omega, x(\omega)) d \mu(\omega)$ (if the integral is not defined then we set $\left.I_{f}(x)=+\infty\right)$.

THEOREM 5.1. If $\mathrm{f}: \Omega \times \mathrm{X} \rightarrow \overline{\mathrm{R}}$ is a normal integrand s.t.

1) there exist $x_{0}(\cdot) \varepsilon L_{x}^{1}$ s.t. $I_{f}\left(x_{0}\right)<\infty$,

b) $I_{f}(\cdot)$ is $w\left(L_{X}^{1}, L_{X^{*}}^{\infty}\right)$-1 ower semi continuous,

then $f(\omega,$.$) is \mu-a . e$ convex

PROOF. Let $E: \Omega+2^{X} \cdot\{\varnothing\}$ be the multifunction definted by $E(\omega)=\operatorname{ep} 1 f(\omega,$.$) .$ Since the integrand $\mathrm{f}(.,$,$) is normal \mathrm{E}($.$) is closed valued and measurable. Also note$ that $\left(x_{0}(\cdot), f^{+}\left(\cdot, x_{0}(\cdot)\right) \varepsilon S_{E}^{1} \neq / 0\right.$. We claim that $S_{E}^{1}$ is w-closed in $L_{X \times R}^{1}(\Omega)$. So let $\left(x_{b}, \lambda_{b}\right) \stackrel{w-L_{X \times R}^{1}}{\longrightarrow}(x, \lambda)$. Then for all $A \varepsilon \Sigma$ we have:

$$
I_{f}^{A}\left(x_{b}\right)=\int_{A} f\left(\omega, x_{b}(\omega)\right) d \mu(\omega)<\int_{A} \lambda_{b}(\omega) d \mu(\omega)
$$

Note that $I_{f}^{A}(z)=I_{f}\left(x_{A} z+x_{A} c^{x_{0}}\right)-\int_{A} f\left(\omega, x_{0}(\omega)\right) d \mu(\omega)$ and $\int_{A} f\left(\omega, x_{0}(\omega)\right) d \mu(\omega)<\infty . \quad A l s 0 z(\cdot)+\left(x_{A} z+x_{A} c_{0} x_{0}\right)$ is affine continuous. So $z \rightarrow I_{f}^{A}(z) 18 w-1.8 . c$. Hence $I_{f}^{A}(x)<\lim I_{f}^{A}\left(x_{b}\right)<\int_{A} \lambda(\omega) d \mu(\omega) \Rightarrow f(\omega, x(\omega))<\lambda(\omega) \mu-a_{\bullet} e_{\bullet} \Rightarrow \quad=>(x, \lambda) \in S_{E}^{1}$ So indeed $\mathrm{S}_{\mathrm{E}}^{1}$ is $\mathrm{w}$-closed in $\mathrm{L}_{\mathrm{XXR}}^{1}$. Applying Lemma $A$ we get that $E(\omega) \in P_{f c}(X) \mu-a . e=z f(\omega,)$.18 pra.e. convex.

Now we pass to the subdifferential of $I_{f}(\cdot)$. So assume that $(\Omega, \Sigma, \mu)$ is a complete, $\sigma$-finite, measure space and $X$ a separable Banach space. We will need the decomposition theorem for $\left[\mathrm{L}_{X}^{\infty}\right]^{\star}$. This result was first proved by Yosida-Hewitt [32] for $X=R$ (see also Rockafellar [27]). Then 1t was extended to separable Banach spaces by Ioffe-Levin [10] and Rockafellar [28] and later to nonseparable Banach space by 
Levin [15]. A functional $u(\cdot) \varepsilon\left[L_{X}^{\infty}\right]^{*}$ is sald to be absolutely continuous with respect to $\mu$, if there exists $g \in L_{X_{w}^{*}}^{1} s \cdot t$.

$$
\left.\langle u, x\rangle=\int_{i s}(g(\omega)), x(\omega)\right) d \mu(\omega) \text { for all } x(\cdot) \varepsilon L_{x^{\bullet}}^{\infty}
$$

A functional $v \varepsilon\left[L_{X}^{\infty}\right]^{\star}$ is said to be singular with respect to $\mu$ if there exist $\left\{\Omega_{n}\right\}_{n>1} \leq \sum$ s.t.

1) $\Omega_{n+1} \subseteq \Omega_{n} n \geqslant 1 \quad$ 1i) $\mu\left(\Omega_{n} \cap A\right) \rightarrow 0$ for all $A \in \Sigma, \mu(A)<\infty$ and

11i) $\langle v, x\rangle=0$ for all $x \varepsilon_{x}^{\infty}$ s.t. $\left.x\right|_{\Omega_{n}}=0$ for some $n>1$.

THEOREM 5.2. [15] Every functional ye[L $\left.\mathrm{L}_{\mathrm{X}}^{\infty}\right]^{\star}$ admits a unique decomposition

$$
y=y_{a}+y_{s} \text { where } y_{0} \text { is absolutely continuous and } y_{s} \text { is singular with }
$$

respect to $\mu$. Furthermore $\|y\|=\left\|y_{0}\right\|+\left\|y_{0}\right\|$.

THEOREM 5.3. If $f: S i x X+\bar{R}$ is a convex, normal integrand and $I_{f}($.$) is strongly$ continuous on $\mathrm{L}_{\mathrm{X}}^{\infty}$ at $\mathrm{x}_{0}(\cdot)$

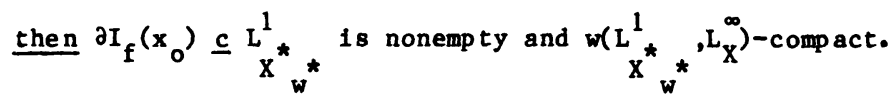

Furthermore $1 f X^{*}$ is separable then $\omega \rightarrow \partial f\left(\omega, x_{0}(\omega)\right)$ is a $P_{w k c}(X)$-valued integrably bounded multifunction.

PROOF. Let $x^{*} \varepsilon\left[L_{X}^{\infty}{ }^{*}\right.$ and 1 et $x^{*}=\left.\left.x^{*}\right|^{+x^{*}}\right|_{s}$ be 1 ts decomposition according to Theorem 5.2. From Levin [15], Theorem 6.4, we know that:

$$
\left(I_{f}\right)^{\star}\left(x^{*}\right)=I_{f *}\left(x_{a}^{*}\right)+\sigma\left(x_{g}^{*}, \operatorname{domI}_{f}\right)
$$

Since $I_{f}(\cdot)$ is s-continuous at $x_{0}, \partial I_{f}\left(x_{0}\right) \neq \emptyset$.

Let $x^{*} \varepsilon \partial I_{f}\left(x_{0}\right)$. Then by definition we have:

$$
\left\langle x_{a}^{*}+x_{s}^{*}, x_{0}\right\rangle=I_{f}\left(x_{0}\right)+\left(I_{f}\right) *\left(x^{*}\right)=I_{f}\left(x_{0}\right)+I_{f *}\left(x_{a}\right)+\sigma\left(x_{s}^{*}, \operatorname{domI} f_{f}\right)
$$

Note that because of the continuity of $I_{f}(\cdot)$ at $x_{0}, x_{0} \varepsilon$ intdom $I_{f}$ and 80 if $x_{s}^{*} \neq 0$ then

$$
\left\langle x_{s}^{*}, x_{0}\right\rangle\left\langle\sigma\left(x_{s}^{*}, \operatorname{dom}_{f}\right)\right.
$$

which when used back in (4.4) produces a contradiction. Therefore $x_{8}^{*}=0 \Rightarrow x^{*}=x_{a}^{*}=\Rightarrow \partial I_{f}\left(x_{0}\right) \subseteq L_{X^{*}}^{1} \cdot$ 
Also the continuity at $x_{0}\left(_{\cdot}\right)$ tells us that $\partial I_{f}\left(x_{0}\right)$ is w*-compact in $\left[L_{x}^{\infty}\right]^{*}$. But the restriction of the $w^{*}-t o p o l o g y$ on $L_{X^{*}}^{1}$ coincides with the $w\left(L_{X}^{1}{ }_{w}{ }^{1}, L_{X}^{\infty}\right)$-topology. Therefore $\partial I_{f}\left(x_{0}\right)$ is $w\left(L_{X}^{1}{ }_{w}, L_{X}^{\infty}\right)$-compact. Now, if $X^{\star}$, is separable, then $L_{X^{*}}^{1}{ }_{w^{*}}=L_{X^{*}}^{1}$ (see Ionescu-Tulcea [11]).

Also from Rockafellar [28], we know that $\partial I_{f}\left(x_{0}\right)=s_{\partial f\left(\cdot, x_{0}(\cdot)\right)}^{1}$. So apply ing proposition 5.1 of [25], we conclude that $\omega+\partial f\left(\omega, x_{0}(\omega)\right)$ is $P_{w k c}(X)$-valued integrably bounded multifunction.

Q.E.D.

REMARK. Our result generalizes Theorem 2 and its corollary in Bismut [3]. In this pape $\mathrm{X}$ was assumed to be separable, reflexive.

Next we look at some special type of subgradients namely extremal subgradients. This spaces are as above.

THEOREM 5.4. If $f: s 2 x X \rightarrow \bar{R}$ is a convex, normal integrand and $\left.I_{f} f_{\bullet}\right)$ is strongly continuous on $\mathrm{L}_{\mathrm{X}}^{\infty}$ at $\mathrm{x}_{\mathrm{o}}(\cdot)$, then $\operatorname{ext} \partial \mathrm{I}_{\mathrm{f}}\left(\mathrm{x}_{0}\right) \neq 0$ and for all ueext$\partial \mathrm{I}_{\mathrm{f}}\left(\mathrm{x}_{0}\right)$, $u(\omega) \varepsilon \operatorname{ext} \partial f\left(\omega, x_{0}(\omega)\right)$ wa.e.

PROOF. We know that $\partial I_{f}\left(x_{0}\right)$ is $w\left(L_{x^{*}}^{1}{ }_{w}^{*}, L_{X}^{\infty}\right)$-compact. So by the Krein-Milman theorem we have that $\operatorname{ext} \partial I_{f}\left(x_{0}\right) \neq 0$. Also $\partial I_{f}\left(x_{0}\right)=s_{\partial f}^{1}\left(\cdot, x_{0}\left(_{0}\right)=\operatorname{ext} \partial I_{f}\left(x_{0}\right)\right.$ $=\operatorname{exts}_{\partial f\left(\cdot, x_{0}(\cdot)\right)}^{1}$. But from Benamara [2], exts ${ }_{\partial f\left(\cdot, x_{0}(\cdot)\right)}^{1}=s_{\operatorname{ext} \partial f\left(\cdot, x_{0}(\cdot)\right)^{\bullet}}^{1}$ Hence $u \in S_{\operatorname{ext} \partial f\left(., x_{0}(\cdot)\right)}^{1} \Rightarrow u(\omega) \operatorname{\varepsilon ext} \partial f\left(\omega, x_{0}(\omega)\right) \mu-a \cdot e$. Q.E.D.

Now we turn our attention for the conjugate of the convex integral functional $I_{f}: L_{X}^{1}+\bar{R}$.

Assume that $(\Omega, \Sigma, \mu)$ is a complete, finite, nonatomic measure space and $X$ a separable Banach space. Recall that $f: X \rightarrow R U\{+\infty\}$ is w-inf-compact, if for all $\lambda \varepsilon R,\{x \in X: f(x)<\lambda\}$ is $w$-compact.

THEOREM 5.5. If $\mathrm{f}: \Omega \times \mathrm{XX} \rightarrow \overline{\mathrm{R}}$ is a convex, norma 1 integrand which is w-inf compact in $x$ for all $\omega \varepsilon \Omega$ and there exists $x(\cdot) \varepsilon L_{X}^{1}$, s.t. $I_{f}(x(\cdot))<+\infty$,

topology). then $I_{f^{*}}(\cdot)$ is $m\left(L_{X^{*}}^{\infty}, L_{W^{*}}^{1}\right)$-continuous (Here $m(.,$.$) ) denotes the Mackey$

PROOF. Since by hypothesis $\mathrm{I}_{\mathrm{f}}$ is w-inf-compact, from Moreau's theorem (see Laurent [14]) we have that $\left(I_{f}\right)^{*}$ is m-continuous at 0 . Also $\left[I_{f}\right]^{*}=I_{f}$ (see Levin [15] or Rockafellar [28]). Since from convex analysis we know that $I_{f *}(\cdot)$ is $\mathrm{m}-$ continuous in the interior of its domain, we have to show that $I_{f *}(\cdot)$ is finite everywhere.

From the fact that $I_{f^{*}}(\cdot)$ is m-continuous at 0 , we get that there exists 
$V \varepsilon N_{m}(0)=\left\{\right.$ filter of $m\left(L_{X_{W^{*}}^{*}}^{\infty}, L_{X}^{1}\right)$-neighborhoods of the origin\} s.t. for all $x^{*} \varepsilon V$ we have

$$
I_{f \star}\left(x^{\star}\right)<I_{f \star}(0)+1
$$

From the definition of the Mackey topology, we know that $V$ is the polar set of a relatively $w$-compact set $W$ in $L_{X}^{1}$. So we can write:

$$
V=\left\{x^{*}(.) \varepsilon \mathrm{L}_{\mathrm{X}^{*}{ }_{w^{*}}^{\infty}}^{\infty}: \sup _{\mathrm{u} \in W} \int_{\Omega}\left(x^{*}(\omega), \mathrm{u}(\omega)\right) \mathrm{d} \mu(\omega)<1\right\}
$$

Since $W$ is relatively w-compact in $L_{X}^{1}$, from Theorem 4, p. 104 of Dieste1-Uh1 [7], we have that $W$ is uniformly integrable. So for all $\varepsilon>0$ there exists $\delta>0$ s.t. if $\mu(A)<\delta$, then $\sup _{u \in W} \int_{A} \| u(\omega)|| d \mu(\omega)<\varepsilon$. Let $x^{*} \varepsilon L_{X_{w^{*}}^{\infty}}^{\infty}, x^{*} \neq \phi$. Take $\varepsilon=\frac{1}{\left\|x^{*}\right\|_{\infty}}$. Then $\sup _{\text {uøW }} \int_{A}\left(x^{*}(\omega), u(\omega)\right) d \mu(\omega)<1$, for $\mu(A)<\delta$. Because $(\Omega, \Sigma, \mu)$ is finite, nonatomic, from Saks lemma we known that we can find $\left\{A_{k}\right\}_{k=1}^{n} \subseteq{ }^{2}$.

$$
\mathrm{U}_{k=1}^{n} A_{k}=\Omega \text { with } \mu\left(A_{k}\right)<\delta \text {, for all } k=1, \ldots, n \text {. Then }
$$

w). $\int_{A_{k}} f^{*}\left(\omega, x^{*}(\omega)\right) d \mu(\omega)=\int_{\Omega} f^{*}\left(\omega, x_{A_{k}}(\omega) x^{*}(\omega)\right) d \mu(\omega)-\int_{A_{k}^{*}}^{c}(\omega, 0) d \mu($ since $I_{f^{*}}($.$) is m-continuous at 0, \int_{A_{k}} f^{\star}(\omega, 0) d \mu(\omega)<\infty$.

Also $\sup _{u \in W} \int_{\Omega}\left(x_{A_{k}}(\omega) x *(\omega), u(\omega)\right) d \mu(\omega)=\sup \int_{u \in W}\left(x_{k}^{*}(\omega), u(\omega)\right) d \mu(\omega)<1$.

Therefore $x_{A_{k}} x^{*}(\cdot) \in V$ and so:

$$
\begin{aligned}
& \int_{\Omega} f^{\star}\left(\omega, x_{A_{k}}(\omega) x^{\star}(\omega)\right) d \mu(\omega)=I_{f^{*}}\left(x_{A_{k}} x^{*}\right)<I_{f^{*}}(0)+1 \\
\Rightarrow & \int_{A_{k}} f^{\star}\left(\omega, x^{\star}(\omega)\right) d \mu(\omega)<+\infty \\
\Rightarrow & I_{f *}\left(x^{*}\right)<\infty
\end{aligned}
$$

and since $\mathrm{x}^{\star} \varepsilon \mathrm{L}_{\mathrm{X}^{\star}}^{\infty}$ was arbitrary, we have that $\operatorname{dom} \mathrm{I}_{\mathrm{f}^{\star}}=\mathrm{L}_{\mathrm{X}^{\star}}^{\infty}{ }_{\mathrm{w}^{\star}}$

Q.E.D.

Now we will obtain a description for $\overline{\operatorname{domI}_{f *}}$ So assume that $(\Omega, \Sigma, \mu)$ is a complete, $\sigma$-finite measure space and $X$ a separable Banach space.

We recall that if $\mathrm{f}: \mathrm{X} \rightarrow \mathrm{RU}\{+\infty\}, \mathrm{f} \neq+\infty$ is convex, then the recession function

$f: X \rightarrow R U\{+\infty\}$ is defined by $f_{\infty}(h)=\sup \{f(x+h)-f(x): x \in \operatorname{domf}\}$. If $f($.$) is in$

addition lower semi continuous, then $f_{\infty}(h)=\sup _{\lambda>0} \frac{f(x+\lambda h)-f(x)}{\lambda}$ 
THEOREM 5.6. If $\mathrm{f}: \Omega \times \mathrm{X}+\overline{\mathrm{R}}$ is a convex, normal integrand, there exist

$x \in L_{X}^{1}$ and $x^{*} L_{X^{*}}^{\infty}$ s.t. $I_{f}(x)<\infty$ and $I_{f^{*}}\left(x^{*}\right)<+\infty$ and $I_{f^{*}}(\cdot)$ is lower semicontinuous

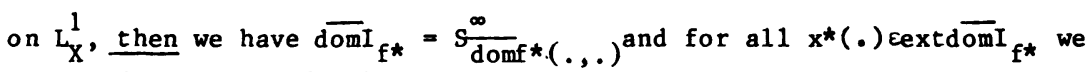
have $x^{*}(\omega) \varepsilon \operatorname{ext} \operatorname{domf} *(\omega,.) \mu-$ a.e.

PROOF. Since by hypothesis $\left.\mathrm{I}_{\mathrm{f}} \mathrm{C}_{\bullet}\right)$ is $1.8 . \mathrm{c}_{\text {. }}$ on $\mathrm{L}_{\mathrm{X}}^{1}$ and convex, from Theorem 6.8 .5 . of Laurent [14], we have:

$$
\left(I_{f}\right)_{\infty}(x)=\sigma\left(x, \operatorname{dom}\left(I_{f}\right) *\right)
$$

Since by hypothesis $\operatorname{dom} I_{f} \neq \emptyset,\left(I_{f}\right) *=I_{f *}$, while from a simple application of the monotone convergence theorem (see also Bismut [3], Proposition 1) we have that:

$$
\left(I_{f}\right)_{\infty}(x)=I_{f_{\infty}}(x)
$$

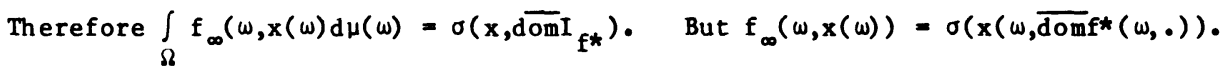
Observe that $\operatorname{domf} *(\omega,)=.U\left\{x^{\star} \varepsilon X^{*}: f^{\star}\left(\omega, x^{*}\right)<n\right\}=m>\omega+\operatorname{domf}(\omega,$.$) is graph$ $n>1$

measurable. Also $x^{\star} \varepsilon S_{\mathrm{domf}^{\star}}^{\infty}(.,$.$) . So applying Theorem 2.2$ of H1a1-Umegak1 [9] we get that:

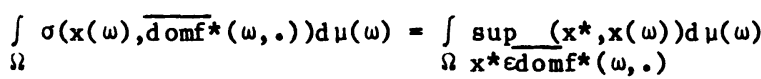

$$
\begin{aligned}
& =\sup \quad \int\left(x^{*}(\omega), x(\omega)\right) d \mu(\omega) \\
& x^{*}(.) \varepsilon S_{\overline{\operatorname{domf}}} *(., .) \\
& =\Rightarrow \sigma\left(x, \overline{\operatorname{domI}}_{f^{\star}}\right)=\sigma\left(x, s_{\overline{\operatorname{domf}}}^{\infty}(., .)\right)
\end{aligned}
$$

Since both sets are clearly convex, we conclude that:

$$
\overline{\operatorname{domI}}_{f *}=s_{\operatorname{domf}}^{\infty}(,, .)
$$

The second part of the conclusion, follows from the following equalities (see Benamara [2]):

$$
\operatorname{ext} \overrightarrow{\operatorname{domI}}_{f *}=\operatorname{exts} \overline{\operatorname{domf}}^{\infty}(,,)=s_{\operatorname{ext} \overline{\operatorname{dom} f} *(., .)}^{\infty}
$$


ACKNOWLEDGEMENT. This research was supported by N.S.F. Grants D.M.S. -8602313 and D.M.S. -8802688 .

\section{REFERENCES}

1. AUMANN, R. Measurable utility and the measurable cholce theorem, La Decision, Colloque International du C.N.R.S.,Paris (1967), 15-26.

2. BENAMARA, M. Sections mesurables extremales d'une multi-application. C.R. Acad. Sc. Paris, t.278 (1974), 1249-1252.

3. BISMUT, J.M. Integrales Convexes et Probabilites, J. Math. Anal. Appl. 42 (1973), 639-673.

4. BOURGAIN, J. An averaging result for $\ell^{l}$-sequences and applications to weakly conditionally compact sets in $\mathrm{L}_{X}^{1}$, Israel J. Math. 32 (1979), 289-298.

5. CASTAING, C. and VALADIER, M. Convex Analysis and Measurable Multifunctions. Lecture Notes in Math, 580 Springer, Ber1in, (1977).

6. COSTE, A. La propriete de Radon-Nikodym en integration multivoque. C.R. Acad. Sc. Paris t.280 (1975), 1515-1518.

7. DIESTEL, J. and UHL, J.J. Veçtor Measures, Math. Surveys, 15, Providence, RI, (1977).

8. HERMES, $H$. and LASALLE, J. Functional Analysis and Time Optimal Control, Academic Press, New York (1969).

9. HIAI, F. and UMEGAKI, H. Integrals, conditional expectations and martingales of multivalued functions. J. Multiv. Ana1. 7 (1977), 149-182.

10. IOFFE, A. and LEVIN, V. Subdifferentials of convex functions, Trans. Moscow Math.Soc. 26 (1972) 3-74.

11. IONESCU-TULCEA, A. and IONESCU-TULCEA, C. Topics in the theory of Liftings, Springer, Berlin (1969).

12. KATO, T. Linear evolution equations of hyperbolic type. I.Fac. Sc. Univ. Tokyo Sec. I. 17 (1970) 241-258.

13. KLEIN, E. and THOMPSON, A. Theory of Correspondences, John Wiley, New York (1984).

14. LAURENT, P.J. Approximation et Optimisation, He rmann, Paris (1972).

15. LEVIN, V. Convex Integral functionals and the theory of lifting, Russian Math. Surveys 30 (1975), 119-184.

16. LINDENSTRAUSS, J. and TZAFRIRI, L. Classical Banach Spaces II: Function Spaces Springer, Berlin (1979).

17. MUSIAL, $K$. and NARDZEWSKI, C.R. Liftings of vector measures and their applications to RNP and WRNP. Lecture Notes in Math. 645, Springer, Berlin (1977).

18. PAPAGEORGIOU, N.S. On the theory of Banach space valued multifunctions.

Part. 1: Integration and conditional expectation. J. Multiv. Ana 1. 17 (1986), 185-206. 
19. PAPAGEORGIOU, N.S. On the theory of Banach space valued multifunctions. Part 2: Set valued martingales and set valued measures. J. Multiv. Ana1. 17 (1986), 207-227.

20. PAPAGEORGIOU, N.S. Efficiency and optimality in economies described by coalitions. J. Math. Ana 1. App1. 116, (1986), 497-512.

21. PAPAGEORGIOU, N.S. Trajectories of set valued integrals. Bull. Austr. Math. Soc. 31 (1985), 389-411.

22. PAPAGEORGIOU, N.S. Representation of set valued operators, Trans. Amer. Math. Soc. 292 (1985), 557-572.

23. PAPGEORGIOU, N.S. On the properties of the Aumann integral, with applicattions to differential inclusions and control systems. Czechoslovak Math. Journ. To appear.

24. PAPAGEORGIOU, N.S. Random fixed point theorems for measurable multifunctions in Banach spaces. Proc. Amer. Soc. 97 (1986), 507-514.

25. PAPAGEORGIOU, N.S. Contributions to the theory of set valued functions and set valued measures. Trans. Amer. Math. Soc. 304 (1987), 245-265.

26. PAPAGEORGIOU, N.S. On the properties of multimeasures with values in a Banach space. submitted.

27. ROCKAFELLAR, R.T. Weak compactness of level sets of integral functionals. Proc. of 3rd Coll. Ana 1. Fonct. (CBRM) H.G. Garnir ed, Vender, Lourain (1971), 85-98.

28. ROCKAFELLAR, R.T. Convex integral functionals and duality in Contributions to Non 1. Ana 1. E. Zarantone110 ed, Acad. Press, New York (1971), 215-236.

29. ROSENTHAL, H. A characterization of Banach spaces containing $\ell^{1}$. Proc.Nat. Acad. Sc. 71 (1974), 2411-2413.

30. SAINT-BEUVE, M.F. On the extension of Von Neumann-Aumann's theorem. J. Funct. Ana 1. 17 (1974), 112-129.

31. TALAGRAND, M. Weak Cauchy sequences in $L^{1}(E) \cdot$ Amer. Jour. of Math. 106 (1984), 703-724.

32. YOSIDA, K. and HEWITT, E. Finctely additive measures. Trans. Amer. Math. Soc. $\underline{72}$ (1952), 46-66. 


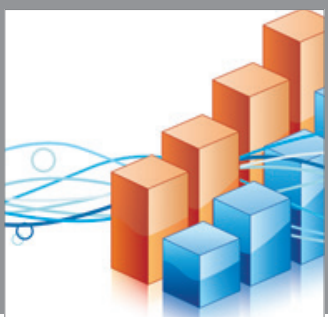

Advances in

Operations Research

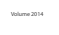

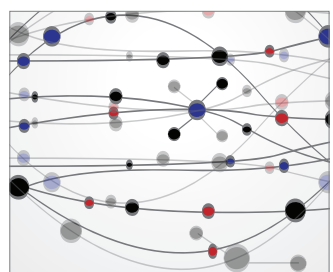

\section{The Scientific} World Journal
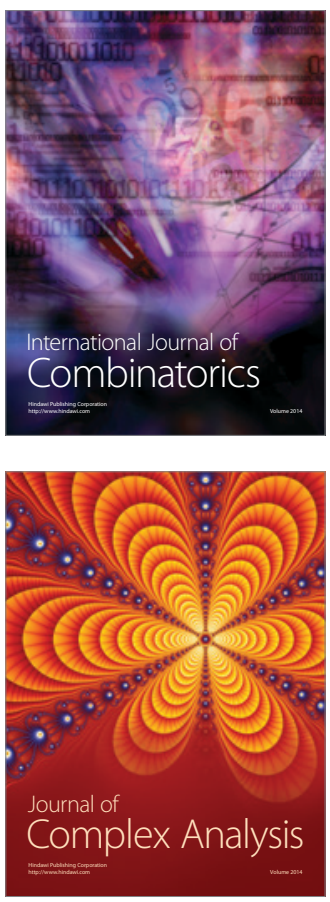

International Journal of

Mathematics and

Mathematical

Sciences
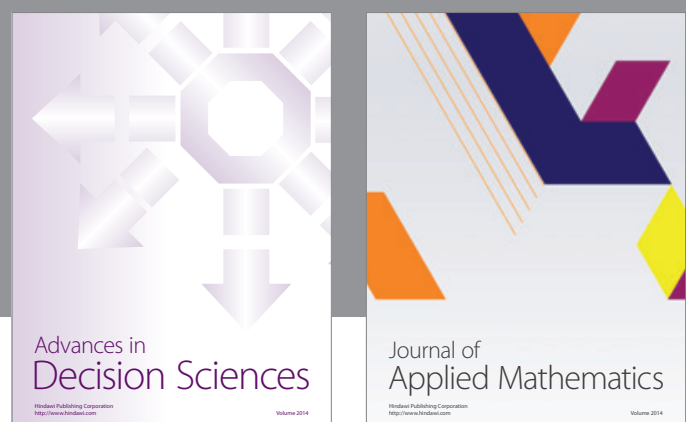

Journal of

Applied Mathematics
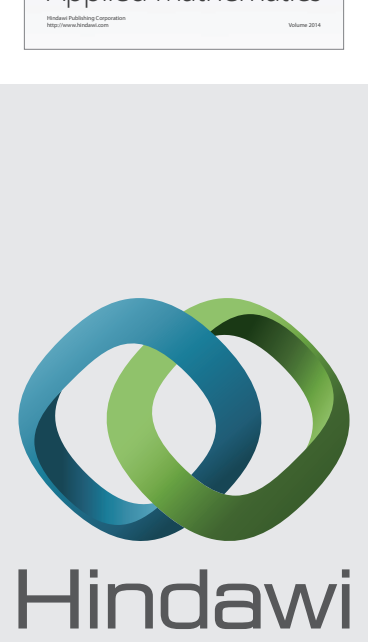

Submit your manuscripts at http://www.hindawi.com
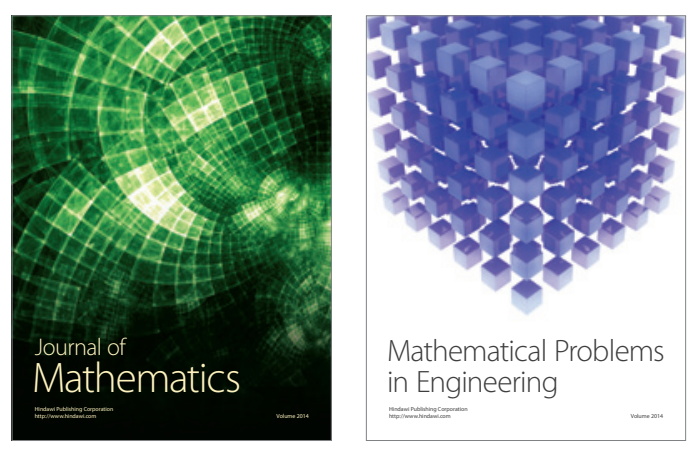

Mathematical Problems in Engineering
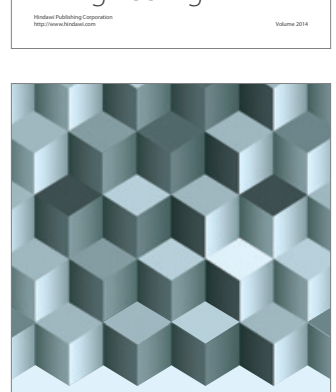

Journal of

Function Spaces
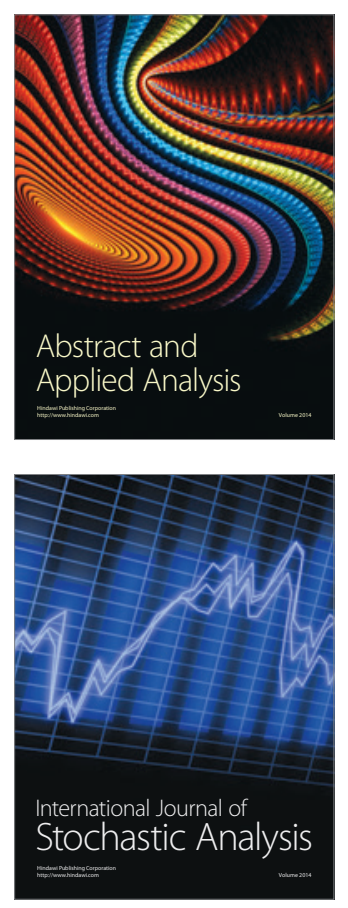

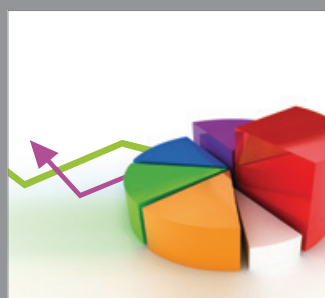

ournal of

Probability and Statistics

Promensencen
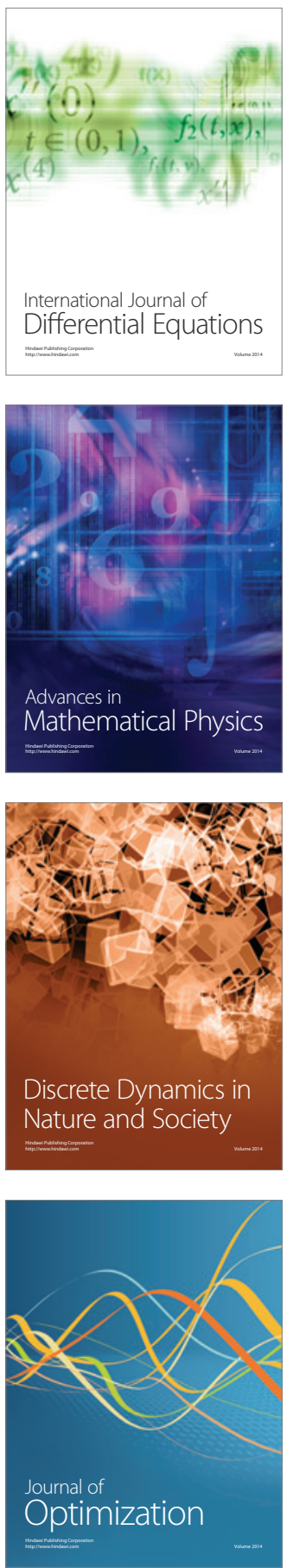